\title{
LINEAR PDES AND NUMERICAL METHODS THAT PRESERVE A MULTI-SYMPLECTIC CONSERVATION LAW
}

\author{
JASON FRANK*, BRIAN E. MOORE ${ }^{\dagger}$, AND SEBASTIAN REICH ${ }^{\ddagger}$
}

Draft version of July 7, 2005

\begin{abstract}
Multi-symplectic methods have recently been proposed as a generalization of symplectic ODE methods to the case of Hamiltonian PDEs. Their excellent long time behavior for a variety of Hamiltonian wave equations has been demonstrated in a number of numerical studies. A theoretical investigation and justification of multi-symplectic methods is still largely missing. In this paper, we study linear multi-symplectic PDEs and their discretization by means of numerical dispersion relations. It is found that multi-symplectic methods in the sense of Bridges \& Reich [7, 19], such as Gauss-Legendre Runge-Kutta methods, possess a number of desirable properties such as non-existence of spurious roots and conservation of the sign of the group velocity. A certain CFL-type restriction on $\Delta t / \Delta x$ might be required for methods higher than second-order in time. It is also demonstrated by means of the explicit midpoint method that multi-step methods may exhibit spurious roots in the numerical dispersion relation for any value of $\Delta t / \Delta x$ despite being multi-symplectic in the sense of discrete variational mechanics [15].
\end{abstract}

1. Introduction. The field of numerical analysis has experienced a recent, as well as growing, interest in geometric integration. When solving differential equations numerically it is important to preserve as much of the qualitative solution behavior as possible. In particular, it is advantageous to numerically preserve the symplectic structure of Hamiltonian systems.

The topic of symplectic integration for Hamiltonian ODEs has been thoroughly analyzed. The concept of symplectic time integration has also been extended to Hamiltonian PDEs (see, e.g., [16]). The concept of space-time multi-symplectic integration is however much more recent and its development is still in the beginning stages. In some respects, even the proper definition of a multi-symplectic integrator is still not clear. The original approach, presented by Marsden, Patrick and Shkoller [15], defines a multi-symplectic method as one which can be derived from a Lagrangian formulation using a discrete variational principle. The original approach was limited to first-order field theories (see [12] for an extension to second-order field theories). The discrete variational approach allows the systematic derivation of one-step and multi-step multi-symplectic methods.

The second approach, which was put forth by Bridges and Reich [7, 19], defines a multisymplectic integrator as a method which exactly preserves a multi-symplectic conservation law by applying a symplectic one-step method such as Störmer-Verlet or implicit midpoint in space and time.

It is our intent to study multi-symplectic integration methods in the context of linear multisymplectic PDEs. Based on a discussion of numerical dispersion relations, we will find that both conservation of a multi-symplectic conservation law and discretization by one-step methods in space and time should be considered essential ingredients of a multi-symplectic method.

According to Bridges [2, 3], a PDE of the general form

$$
\mathbf{K} \mathbf{z}_{t}+\mathbf{L} \mathbf{z}_{x}=\nabla_{\mathbf{z}} S(\mathbf{z})
$$

is called multi-symplectic provided $\mathbf{K}$ and $\mathbf{L}$ are (constant) $d \times d$ skew-symmetric matrices, and $S$ : $\mathbb{R}^{d} \rightarrow \mathbb{R}$ is a smooth function. Here and throughout, subscripts are used to denote differentiation in the standard way, and we define $\mathbf{z}=\mathbf{z}(x, t)$ to be a $d$-dimensional vector of state variables. Many Hamiltonian PDEs can be formulated in this way, and the multi-symplectic formulation has become useful for understanding these problems (cf. $[2,3,4,5,6,8]$ ).

${ }^{*}$ CWI, P.O. Box 94079, 1090 GB Amsterdam, The Netherlands (e-mail: jason@cwi.nl). Supported by an Innovational Research grant from the Netherlands Organization for Scientific Research (NWO)

$\dagger$ Department of Mathematics and Statistics, McGill University, Montreal, Canada (e-mail: moore@math.mcgill.ca)

${ }_{\ddagger}^{\ddagger}$ Institut für Mathematik, Universität Potsam, Postfach 6015 53, D-14415 Potsdam, Germany (e-mail: sreich@math.uni-potsdam.de) 
As one would expect, however, the nonlinearity of equation (1.1) makes the study of numerical solutions for the problem more difficult, and there is still much to understand with regard to multisymplectic discretization methods. Hence, the aim of this paper is to conduct an extensive analysis of multi-symplectic discretization methods for the linear equation

$$
\mathbf{K} \mathbf{z}_{t}+\mathbf{L} \mathbf{z}_{x}=\mathbf{A z}
$$

where $\mathbf{A}$ is symmetric. This allows one to examine solutions and dispersion relations for both the continuous and discrete versions of this equation, providing a thorough understanding of the behavior of our numerical methods for linear problems.

We start by making the standard (complex-valued) separation of variables ansatz

$$
\mathbf{z}(x, t)=u(t) v(x) \mathbf{a}
$$

where $u(t)$ and $v(x)$ are complex-valued functions and $\mathbf{a}$ is a $d$-dimensional complex-valued vector. Substituting this ansatz into the linear PDE (1.2) leads to the linear system

$$
\left(\frac{u^{\prime}}{u} \mathbf{K}+\frac{v^{\prime}}{v} \mathbf{L}-\mathbf{A}\right) \mathbf{a}=\mathbf{0}
$$

Solutions are found by setting

$$
\lambda=\frac{u^{\prime}}{u} \quad \text { and } \quad \mu=\frac{v^{\prime}}{v}
$$

with the complex numbers $\lambda$ and $\mu$ satisfying

$$
\operatorname{det}(\lambda \mathbf{K}+\mu \mathbf{L}-\mathbf{A})=0 .
$$

Since we are interested in wave-like solutions we furthermore seek (complex-valued) solutions with $\lambda=i \omega$ and $\mu=i \kappa$, i.e.

$$
\mathbf{z}(x, t)=\mathrm{e}^{\mathrm{i}(k x+\omega t)} \mathbf{a} .
$$

Here, $k$ denotes the wave number and $\omega$ denotes the wave frequency, which must satisfy the dispersion relation $[3]$

$$
\mathcal{D}(\omega, k):=\operatorname{det}(\mathbf{i} \omega \mathbf{K}+\mathbf{i} k \mathbf{L}-\mathbf{A})=0 \text {. }
$$

It is important to note that the matrix used in this calculation is self-adjoint, i.e., $\overline{\mathcal{D}(\omega, k)}=$ $\mathcal{D}(\bar{\omega}, \bar{k})$, from which it is immediate that $0=\mathcal{D}(\omega, k)=\mathcal{D}(\bar{\omega}, k)$ for real $k$. Hence, for any given real $k$, solutions $\omega$ of the dispersion relation are either real or come in complex-conjugate pairs meaning there is no diffusion [20, Chapter 11].

With the dispersion relation, one can write the frequency as a function of the wave number, such that

$$
\mathcal{D}(\omega, k)=0 \quad \Longleftrightarrow \quad \omega=\omega(k),
$$

at least locally. Then, depending on the function $\mathcal{D}$, there may be multiple frequencies $\omega_{i}$ for every $k$, corresponding to different modes. One could also pose the reverse question and ask how many different wave numbers $k$ can give rise to a given frequency $\omega$.

It is worthwhile to note that taking the complex-conjugate in (1.3) also yields a solution and hence

$$
\mathbf{z}(x, t)=\frac{1}{2}\left[\mathbf{a} \mathrm{e}^{\mathrm{i}(k x+\omega t)}+\overline{\mathbf{a}} \mathrm{e}^{-\mathrm{i}(k x+\omega t)}\right]
$$


yields real-valued solutions. The dispersion relation is not restricted to single mode solutions (1.3). In fact, the most general oscillatory solution of the linear PDE (1.2) can be stated as a superposition of solutions

$$
\mathbf{z}(x, t)=\frac{1}{2} \sum_{i} \int_{0}^{\infty} \mathbf{a}_{i}(k) \mathrm{e}^{\mathrm{i}\left(k x-\omega_{i}(k) t\right)} \mathrm{d} k+\frac{1}{2} \sum_{i} \int_{0}^{\infty} \overline{\mathbf{a}}_{i}(k) \mathrm{e}^{-\mathrm{i}\left(k x-\omega_{i}(k) t\right)} \mathrm{d} k, \quad-\infty<x<\infty,
$$

which is obtained through Fourier analysis [20, Chapter 11]. Here the index $i$ runs over all solution branches of the dispersion relation.

An important derived quantity is the group velocity defined by

$$
v_{g}=\frac{d}{d k} \omega(k)=-\frac{\partial \mathcal{D}}{\partial k} / \frac{\partial \mathcal{D}}{\partial \omega} .
$$

The group velocity characterizes the speed of energy transport in wave packets [20, Chapter 11] and, hence, is fundamental to the understanding of linear waves. Following Whitham [20, Chapter 11], we introduce the Lagrangian density

$$
\mathcal{L}=\frac{1}{2} \overline{\mathbf{a}}^{T}[i \omega \mathbf{K}+i k \mathbf{L}-\mathbf{A}] \mathbf{a}
$$

and define the energy density

$$
E=\omega \frac{\partial \mathcal{L}}{\partial \omega}=\frac{i \omega}{2} \overline{\mathbf{a}}^{T} \mathbf{K a}
$$

and the energy flux

$$
F=-\omega \frac{\partial \mathcal{L}}{\partial k}=-\frac{i \omega}{2} \overline{\mathbf{a}}^{T} \mathbf{L a}
$$

It holds that $F=v_{g} E$ since $[2]$

$$
v_{g}=-\frac{\partial \mathcal{L}}{\partial k} / \frac{\partial \mathcal{L}}{\partial \omega}=-\frac{\overline{\mathbf{a}}^{T} \mathbf{L a}}{\overline{\mathbf{a}}^{T} \mathbf{K a}}
$$

We wish to extend the linear dispersion analysis to multi-symplectic integration methods. As multi-symplectic methods are obtained as a space-time concatenation of symplectic methods, we start the discussion with a review of results for symplectic ODE methods.

2. Symplectic Integration of Hamiltonian ODEs. We recall some basic properties of symplectic integration methods for Hamiltonian ODEs of the form

$$
\mathbf{K} \mathbf{z}_{t}=\nabla_{\mathbf{z}} S(\mathbf{z})
$$

where $\mathbf{K}$ is a (constant) $d \times d$ skew-symmetric matrix and $S: \mathbb{R}^{d} \rightarrow \mathbb{R}$ a smooth function. A numerical one-step method

$$
\mathbf{z}^{n+1}=\Psi_{\Delta t}\left(\mathbf{z}^{n}\right)
$$

is called symplectic if

$$
\mathbf{d} \mathbf{z}^{n+1} \wedge \mathbf{K} \mathbf{d} \mathbf{z}^{n+1}=\mathbf{d} \mathbf{z}^{n} \wedge \mathbf{K} \mathbf{d} \mathbf{z}^{n}
$$

(See [13] for details and use of wedge product notation.) Let us discuss implications for the linearized equations

$$
\mathbf{K} \mathbf{z}_{t}=\mathbf{A} \mathbf{z} \text {, }
$$


where $\mathbf{A}$ is symmetric. We assume that the one-step method (2.2) is a Runge-Kutta (RK) method with stability function $R(z)$ [11]. Then the (complex-valued) numerical solution of (2.4) can be written in the form

$$
\mathbf{z}^{n}=\sum_{i} R\left(\lambda_{i} \Delta t\right)^{n} \mathbf{a}_{i}
$$

where $\lambda_{i}$ and $\mathbf{a}_{i}$ have to satisfy the generalized eigenvalue problem

$$
\left[\lambda_{i} \mathbf{K}-\mathbf{A}\right] \mathbf{a}_{i}=0
$$

associated with (2.4). Note that if $\lambda$ is an eigenvalue of (2.5) then $-\lambda$ is also an eigenvalue. Furthermore, a symplectic RK method satisfies $R(\lambda) R(-\lambda)=1$. The same property holds for all (not necessarily symplectic) symmetric RK methods [11].

If we now restrict the analysis to the symplectic Gauss-Legendre Runge-Kutta (GL RK) methods, then we conclude from $R(\lambda) R(-\lambda)=1$ and A-stability of the method that $R(z)$ is on the unit circle for purely imaginary valued $\lambda=i \omega$. We write this in the form

$$
R(\mathrm{i} \omega \Delta t)=\mathrm{e}^{\mathrm{i} \phi}
$$

with an appropriate phase function $\phi(\omega \Delta t)$ continuous in $\omega \Delta t$.

Let us explicitly derive the phase function $\phi$ for the implicit midpoint rule

$$
\mathbf{K} \frac{\mathbf{z}^{n+1}-\mathbf{z}^{n}}{\Delta t}=\mathbf{A} \frac{\mathbf{z}^{n+1}+\mathbf{z}^{n}}{2} .
$$

We write $\phi=\Omega \Delta t$ and obtain

$$
\frac{\mathrm{e}^{\mathrm{i} \Omega \Delta t}-1}{\Delta t} \mathbf{K} \mathbf{a}_{i}=\frac{\mathrm{e}^{\mathrm{i} \Omega \Delta t}+1}{2} \mathbf{A} \mathbf{a}_{i}
$$

for each solution mode and, using (2.5) with $\lambda_{i}=i \omega_{i}$, this equation implies

$$
\frac{\mathrm{e}^{\mathrm{i} \Omega \Delta t}-1}{\Delta t} \mathbf{K a}=i \omega_{i} \frac{\mathrm{e}^{\mathrm{i} \Omega \Delta t}+1}{2} \mathbf{K a} .
$$

Hence we must have

$$
\frac{\mathrm{e}^{\mathrm{i} \Omega \Delta t}-1}{\Delta t}=\mathrm{i} \omega_{i} \frac{\mathrm{e}^{\mathrm{i} \Omega \Delta t}+1}{2},
$$

which yields the definition for the stability function of the implicit midpoint method, i.e.

$$
\mathrm{e}^{\mathrm{i} \Omega \Delta t}=R(\mathrm{i} \omega \Delta t)=\frac{1+\mathrm{i} \omega \Delta t / 2}{1-\mathrm{i} \omega \Delta t / 2},
$$

and

$$
\phi(\omega \Delta t)=2 \tan ^{-1}(\omega \Delta t / 2) \in(-\pi,+\pi)
$$

is a monotone function in $\omega \Delta t$.

The fourth-order GL RK method has stability function

$$
R(z)=\frac{1+z / 2+z^{2} / 12}{1-z / 2+z^{2} / 12}
$$

Setting $z=i \omega \Delta t$ and $R(i \omega \Delta t)=e^{i \phi}$ results in

$$
\tan \frac{\phi}{2}=\frac{\omega \Delta t}{2-\frac{\omega^{2} \Delta t^{2}}{6}}
$$


For positive $\omega$, the function $\phi(\omega \Delta t)$ can be chosen to increase monotonically from zero for $\omega=0$ to $\phi(\omega \Delta t)=2 \pi$ for $\omega \Delta t \rightarrow \infty$. A similar statement holds for negative frequencies. Of course, from a sampling point of view, we have $\mathrm{e}^{\mathrm{i} \Omega \Delta t}=\mathrm{e}^{\mathrm{i} \phi}$ with

$$
\Omega=\frac{1}{\Delta t}[\bmod (\phi(\omega \Delta t)+\pi, 2 \pi)-\pi] \in(-\pi / \Delta t,+\pi / \Delta t] .
$$

Note that the implicit midpoint method simply yields

$$
\Omega=\frac{1}{\Delta t} \phi(\omega \Delta t)
$$

with phase function $\phi$ defined by (2.6).

For a general $s$-stage GL RK method of order $p=2 s$ it holds that $\phi(\omega \Delta t)$ is monotone with values in the interval $(-s \pi,+s \pi), \phi(-y)=-\phi(y)$, and $\phi(y)<y$ for $y>0$ [10]. Due to sampling on a discrete grid, i.e. (2.7), it can nevertheless happen that there are values of $\Delta t$ for which $\Omega_{i}=\Omega_{j}$ even though $\omega_{i} \neq \omega_{j}$. These numerically induced 1-1 resonances can occur for $s \geq 2$ and

$$
\max _{i}\left|\phi\left(\omega_{i} \Delta t\right)\right|>\pi
$$

Numerical resonance can lead to instabilities of the scheme under small perturbations [13]. Note that the implicit midpoint method and higher-order GL RK methods can be subject to other numerically induced resonance instabilities. See, e.g., Mandziuk and Schlick [14].

Our discussion of GL RK methods applied to (linear) Hamiltonian ODEs may be summarized as follows:

(i) Oscillatory solutions of (2.4) with frequency $\omega$ are represented numerically by an oscillatory solution with frequency $\Omega$.

(ii) There is one-to-one correspondence between numerical and analytic ${ }^{1}$ solutions modes (no spurious computational modes as for linear multi-step methods).

(iii) The phase function $\phi$ is monotone. However numerical resonances due to (2.7) are possible for GL RK methods with more than one stage.

It should be noted that the same statements would be true for any symmetric and A-stable implicit RK method such as Lobatto IIIA and Lobatto IIIB [11]. However the family of Lobatto IIIA and IIIB methods is not symplectic in the sense of $(2.3)$.

We will now investigate how these properties translate to multi-symplectic discretization methods. We start with a discussion of the Preissman box scheme.

3. The Preissman box scheme. Applying the implicit midpoint rule to the PDE (1.1) in both space and time yields,

$$
\mathbf{K} \delta_{t}^{+} \mathbf{z}^{n, m+1 / 2}+\mathbf{L} \delta_{x}^{+} \mathbf{z}^{n+1 / 2, m}=\nabla S\left(z^{n+1 / 2, m+1 / 2}\right),
$$

where we define the edge midpoint approximations

$$
\mathbf{z}^{n+1 / 2, m}=\frac{1}{2}\left(\mathbf{z}^{n+1, m}+\mathbf{z}^{n, m}\right), \quad \mathbf{z}^{n, m+1 / 2}=\frac{1}{2}\left(\mathbf{z}^{n, m+1}+\mathbf{z}^{n, m}\right),
$$

the cell center approximation

$$
\mathbf{z}^{n+1 / 2, m+1 / 2}=\frac{1}{4}\left(\mathbf{z}^{n+1, m+1}+\mathbf{z}^{n+1, m}+\mathbf{z}^{n, m+1}+\mathbf{z}^{n, m}\right),
$$

and the finite difference approximations

$$
\delta_{t}^{+} \mathbf{z}^{n, m+1 / 2}=\frac{1}{\Delta t}\left(\mathbf{z}^{n+1, m+1 / 2}-\mathbf{z}^{n, m+1 / 2}\right), \quad \delta_{x}^{+} \mathbf{z}^{n+1 / 2, m}=\frac{1}{\Delta x}\left(\mathbf{z}^{n+1 / 2, m+1}-\mathbf{z}^{n+1 / 2, m}\right) .
$$

\footnotetext{
${ }^{1}$ The qualifier analytic will be used throughout the paper to refer to a property of the given continuum equations (e.g. analytic solutions, analytic dispersion relation, and analytic group velocity) as opposed to properties of the numerical scheme (e.g. numerical solutions, numerical dispersion relation, and numerical group velocity).
} 
In addition, define

$$
\tau=\frac{\Delta t}{2} \quad \text { and } \quad \chi=\frac{\Delta x}{2}
$$

through the remainder of the text.

Proposition 1. The Preissman box scheme (3.1) exactly preserves a multi-symplectic conservation law

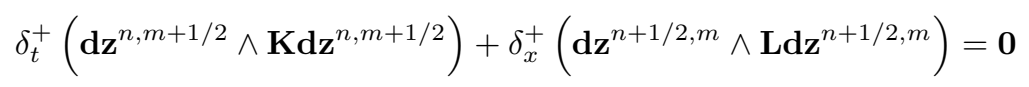

Proof. Proof of this result is due to Bridges and Reich [7].

Applying the Preissman box scheme to the linear PDE (1.2) yields,

$$
\mathbf{K} \delta_{t}^{+} \mathbf{z}^{n, m+1 / 2}+\mathbf{L} \delta_{x}^{+} \mathbf{z}^{n+1 / 2, m}=\mathbf{A} \mathbf{z}^{n+1 / 2, m+1 / 2} .
$$

Based upon the results of $[17,18]$, the modified equation for the linear PDE (1.2) can be stated explicitly as

$$
\mathbf{K}\left(\mathbf{z}_{t}-\frac{\tau^{2}}{3} \mathbf{z}_{t t t}+\frac{2 \tau^{4}}{15} \mathbf{z}_{t t t t t}-\ldots\right)+\mathbf{L}\left(\mathbf{z}_{x}-\frac{\chi^{2}}{3} \mathbf{z}_{x x x}+\frac{2 \chi^{4}}{15} \mathbf{z}_{x x x x x}-\ldots\right)=\mathbf{A} \mathbf{z} .
$$

This is a higher order linear PDE and we may substitute a standard solution ansatz of the form

$$
\mathbf{z}(x, t)=\mathrm{e}^{\mathrm{i}(\Omega t+K x)} \mathbf{a}
$$

into the equation. This yields

$$
\left(\mathbf{K i}\left(\Omega+\frac{\tau^{2} \Omega^{3}}{3}+\frac{2 \tau^{4} \Omega^{5}}{15}+\ldots\right)+\mathbf{L i}\left(K+\frac{\chi^{2} K^{3}}{3}+\frac{2 \chi^{4} K^{5}}{15}+\ldots\right)-\mathbf{A}\right) \mathbf{a}=\mathbf{0} .
$$

Then, due to the identity

$$
\tan (\theta)=\theta+\frac{\theta^{3}}{3}+\frac{2 \theta^{5}}{15}+\ldots
$$

we obtain

$$
\left(\frac{i}{\tau} \tan (\tau \Omega) \mathbf{K}+\frac{i}{\chi} \tan (\chi K) \mathbf{L}-\mathbf{A}\right) \mathbf{a}=\mathbf{0} .
$$

The modified equation can also be stated in closed form because the expansions in (3.3) converge and is given by

$$
\frac{1}{\tau} \mathbf{K} \tanh \left(\tau \partial_{t}\right) \mathbf{z}+\frac{1}{\chi} \mathbf{L} \tanh \left(\chi \partial_{x}\right) \mathbf{z}=\mathbf{A} \mathbf{z} .
$$

Furthermore, the following theorem follows immediately from (3.4).

Theorem 1. The Preissman box scheme (3.2) preserves the analytic dispersion relation of the PDE, such that

$$
\mathcal{D}(\omega, k)=0,
$$

for $\mathcal{D}$ as given in (1.4) with

$$
\omega=\frac{\tan (\tau \Omega)}{\tau} \quad{ }_{6}^{\text {and }} \quad k=\frac{\tan (\chi K)}{\chi},
$$


for $-\frac{\pi}{2}<\tau \Omega<\frac{\pi}{2}$ and $-\frac{\pi}{2}<\chi K<\frac{\pi}{2}$. In other words, the numerical dispersion relation is given by

$$
\mathcal{D}_{N}(\Omega, K):=\mathcal{D}\left(\frac{\tan (\tau \Omega)}{\tau}, \frac{\tan (\chi K)}{\chi}\right)=0
$$

This result was first published by Ascher and McLachlan [1]. See also [17]. Three important conclusions are to be drawn. (i) The numerical group velocity $V_{g}$ is defined by

Since both

$$
V_{g}=\frac{d}{d K} \Omega(K)=-\frac{\partial \mathcal{D}}{\partial k} \frac{\partial k}{\partial K} / \frac{\partial \mathcal{D}}{\partial \omega} \frac{\partial \omega}{\partial \Omega}=v_{g} \frac{\partial k}{\partial K} / \frac{\partial \omega}{\partial \Omega} .
$$

$$
\frac{\partial \omega}{\partial \Omega}>0 \quad \text { and } \quad \frac{\partial k}{\partial K}>0
$$

we have derived

COROLlaRY 1. The sign of the analytic group velocity $v_{g}$ is preserved under the Preissman box scheme.

(ii) The monotonicity and invertibility of (3.6) implies

COROLlaRY 2. The numerical dispersion relation has as many solution branches as the analytic relation and the multiplicity of solutions for fixed $\omega$ and $k$, respectively, is preserved.

(iii) We consider the Lagrangian density (1.6) with $\omega$ and $k$ now functions of $\Omega$ and $K$, respectively, and define the numerical energy density and flux for a single mode solution by

$$
E_{N}=\frac{i \Omega}{2} \frac{\partial \omega}{\partial \Omega} \overline{\mathbf{a}}^{T} \mathbf{K a}, \quad F_{N}=-\frac{i \Omega}{2} \frac{\partial k}{\partial K} \overline{\mathbf{a}}^{T} \mathbf{L} \mathbf{a} .
$$

COROLlaRY 3. The numerical energy density and flux satisfy

$$
F_{N}=V_{g} E_{N}
$$

for a single mode solution, where $V_{g}$ is the numerical dispersion relation of the Preissman box scheme.

Proof. The statement follows from the definitions (3.7) and the total derivative of

$$
\overline{\mathbf{a}}^{T}\left(\frac{i}{\tau} \tan (\tau \Omega) \mathbf{K}+\frac{i}{\chi} \tan (\chi K) \mathbf{L}-\mathbf{A}\right) \mathbf{a}=0
$$

with respect to $K$.

4. Numerical dispersion relation for multi-symplectic GL RK methods. We now discuss the discretization of a linear multisymplectic PDE (1.2) using GL RK methods (possibly of different orders) in space and time. We apply an $s^{0}$-stage GL RK method in time with Butcher arrays $A^{0}=\left(a_{i j}^{0}\right), b^{0}=\left(b_{i}^{0}\right), i, j=1, \ldots, s^{0}$, and a similarly denoted $s^{1}$-stage GL RK method in space.

Reserve the indices $m$ and $n$ for the spatial and temporal meshes, resp.; the indices $i$ and $j$ for spatial and temporal stage indices, resp. The index $k$ is a dummy index. Summation is implied by repeated indices in a product.

Let $\mathbf{Z} \approx \mathbf{z}$ at interior stages, $\boldsymbol{\zeta}, \boldsymbol{\sigma} \approx \boldsymbol{z}$ on horizontal and vertical edges, resp. Let $\mathbf{T} \approx \mathbf{z}_{t}$ at interior stages, $\boldsymbol{\tau} \approx \mathbf{z}_{t}$ on edges. Let $\mathbf{X} \approx \mathbf{z}_{x}$ at interior stages, $\boldsymbol{\xi} \approx \mathbf{z}_{x}$ on edges. Let $\mathbf{U} \approx \mathbf{z}_{x t}$ at interior stages. (See Figure 4.1.)

$$
\begin{aligned}
\mathbf{K} \mathbf{T}_{i j}+\mathbf{L} \mathbf{X}_{i j} & =\mathbf{A} \mathbf{Z}_{i j} \\
\mathbf{X}_{i j} & =\boldsymbol{\xi}_{i}^{n}+\Delta t a_{j k}^{0} \mathbf{U}_{i k} \\
\mathbf{T}_{i j} & =\boldsymbol{\tau}_{j}^{m}+\Delta x a_{i k}^{1} \mathbf{U}_{k j} \\
\mathbf{Z}_{i j} & =\boldsymbol{\zeta}_{i}^{n}+\Delta t a_{j k}^{0} \mathbf{T}_{i k} \\
& =\boldsymbol{\sigma}_{j}^{m}+\Delta x a_{i k}^{1} \mathbf{X}_{k j}
\end{aligned}
$$




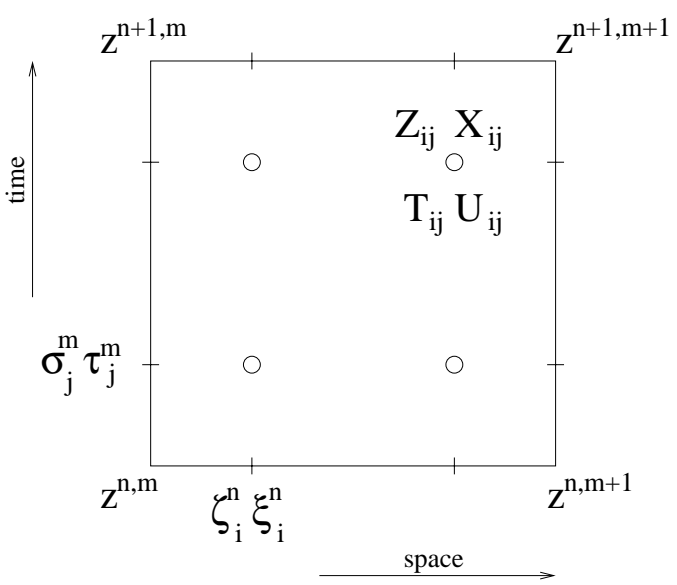

FIG. 4.1. Arrangement of approximate quantities in (4.1)-(4.11).

$$
\begin{aligned}
\boldsymbol{\xi}_{i}^{n+1} & =\boldsymbol{\xi}_{i}^{n}+\Delta t b_{j}^{0} \mathbf{U}_{i j} \\
\boldsymbol{\zeta}_{i}^{n+1} & =\boldsymbol{\zeta}_{i}^{n}+\Delta t b_{j}^{0} \mathbf{T}_{i j} \\
\boldsymbol{\tau}_{j}^{m+1} & =\boldsymbol{\tau}_{j}^{m}+\Delta x b_{i}^{1} \mathbf{U}_{i j} \\
\boldsymbol{\sigma}_{j}^{m+1} & =\boldsymbol{\sigma}_{j}^{m}+\Delta x b_{i}^{1} \mathbf{X}_{i j} \\
\mathbf{z}^{n+1, m+1} & =\mathbf{z}^{n, m+1}+\Delta t b_{j}^{0} \boldsymbol{\tau}_{j}^{m+1} \\
& =\mathbf{z}^{n+1, m}+\Delta x b_{i}^{1} \boldsymbol{\xi}_{i}^{n+1}
\end{aligned}
$$

The equations (4.1), (4.4), (4.5), (4.7) and (4.9) form a closed set (same number of equations as unknowns). To include the grid point data, either $\{(4.2),(4.6)$ and $(4.11)\}$ or $\{(4.3),(4.8)$ and (4.10) $\}$ should be added. The remaining relations are redundant.

We will concentrate on equations (4.1), (4.4), (4.5), (4.7) and (4.9) in the following. Let us assume that we have $\omega, k$ and a satisfying the plane wave solution

$$
[i \omega \mathbf{K}+i k \mathbf{L}-\mathbf{A}] \mathbf{a}=\mathbf{0} .
$$

We look for a (complex-valued) numerical solution of the form

$$
\mathbf{z}^{n, m}=\mathbf{a}^{n m}:=\mathrm{e}^{\mathrm{i} \Omega n \Delta t} \mathrm{e}^{\mathrm{i} K m \Delta x} \mathbf{a} .
$$

Let us therefore make the ansatz that all stage vectors are proportional to $\mathbf{a}^{n m}$ :

$$
\mathbf{Z}_{i j}^{n m}=\gamma_{i j} \mathbf{a}^{n m}, \quad \mathbf{X}_{i j}^{n m}=x_{i j} \mathbf{a}^{n m}, \quad \mathbf{T}_{i j}^{n m}=t_{i j} \mathbf{a}^{n m}, \quad \boldsymbol{\zeta}_{i}^{n}=\alpha_{i} \mathbf{a}^{n m}, \quad \sigma_{j}^{m}=\beta_{j} \mathbf{a}^{n m}
$$

Substituting this solution into (4.1) gives

$$
\left[t_{i j} \mathbf{K}+x_{i j} \mathbf{L}-\gamma_{i j} \mathbf{A}\right] \mathbf{a}^{n m}=0,
$$

for which the dispersion relation yields

$$
\frac{t_{i j}}{\gamma_{i j}}=\mathrm{i} \omega, \quad \frac{x_{i j}}{\gamma_{i j}}=\mathrm{i} k,
$$

that is, $\mathbf{T}_{i j}=i \omega \mathbf{Z}_{i j}$ and $\mathbf{X}_{i j}=i k \mathbf{Z}_{i j}$. 
Next, equation (4.4) yields

$$
\gamma_{i j} \mathbf{a}^{n m}=\alpha_{i} \mathbf{a}^{n m}+i \omega \Delta t a_{j k}^{0} \gamma_{i k} \mathbf{a}^{n m},
$$

For each $i$ we obtain the solution

$$
\left(I-i \omega \Delta t A^{0}\right) \gamma_{i:}=\alpha_{i} 1, \quad \gamma_{i:}=\left(\gamma_{i 1}, \ldots, \gamma_{i s^{1}}\right)^{T}
$$

from which it follows that $\gamma_{i j}=\lambda_{i} \mu_{j}$ with

$$
\boldsymbol{\mu}=\left(I-i \omega \Delta t A^{0}\right)^{-1} 1, \quad \boldsymbol{\alpha}=\boldsymbol{\lambda} .
$$

Similarly, equation (4.5) yields

$$
\boldsymbol{\lambda}=\left(I-i k \Delta x A^{1}\right)^{-1} \mathbb{1}, \quad \boldsymbol{\beta}=\boldsymbol{\mu} .
$$

Finally substitution into (4.7) gives

$$
\lambda_{i}\left(\mathrm{e}^{\mathrm{i} \Omega \Delta t}-1\right) \mathbf{a}^{n m}=i \omega \Delta t b_{j}^{0} \lambda_{i} \mu_{j} \mathbf{a}^{n m},
$$

or,

$$
\left(\mathrm{e}^{\mathrm{i} \Omega \Delta t}-1\right)=\mathrm{i} \omega \Delta t\left(b^{0}\right)^{T} \boldsymbol{\mu}, \quad \Rightarrow \quad e^{\mathrm{i} \Omega \Delta t}=1+\mathrm{i} \omega \Delta t\left(b^{0}\right)^{T}\left(I-\mathrm{i} \omega \Delta t A^{0}\right)^{-1} 11=R^{0}(\mathrm{i} \omega \Delta t) .
$$

And (4.9) similarly yields

$$
\mathrm{e}^{\mathrm{i} K \Delta x}=R^{1}(\mathrm{i} k \Delta x) .
$$

We have effectively proven the following theorem.

TheOREM 2. Let two GL RK methods with stability functions $R^{0}(z)$ and $R^{1}(z)$ be applied to the temporal and spatial derivatives of the linear PDE (1.2), respectively. Given a (complex) plane wave solution (1.3) to (1.2), there exists a numerical plane wave solution of the form

$$
\mathbf{z}^{n, m}=\mathrm{e}^{\mathrm{i} \Omega n \Delta t} \mathrm{e}^{\mathrm{i} K m \Delta x} \mathbf{a}=R^{0}(\mathrm{i} \omega \Delta t)^{n} R^{1}(\mathrm{i} k \Delta x)^{m} \mathbf{a}
$$

where $\omega$ and $k$ satisfy the analytic dispersion relation $\mathcal{D}(\omega, k)=0$. The numerical dispersion relation

$$
\mathcal{D}_{N}(\Omega, K)=0
$$

follows from the analytic one by making use of the relations $\omega(\Omega)$ and $k(K)$ defined implicitly through (4.12) and (4.13). The numerical dispersion relation satisfies

$$
\overline{\mathcal{D}_{N}(\Omega, K)}=\mathcal{D}_{N}(\bar{\Omega}, \bar{K}) .
$$

Since any symmetric and A-stable implicit RK method behaves like a symplectic method on the linear equation level, Theorem 2 also applies to those RK methods as well and Lobatto IIIA and IIIB methods in particular.

We may write $R^{0}(\mathrm{i} y)=\mathrm{e}^{\mathrm{i} \phi(y)}$ and $R^{1}(\mathrm{i} y)=\mathrm{e}^{\mathrm{i} \theta(y)}$, where $\phi$ and $\theta$ are monotone phase functions as defined in $\S 2$. Hence the practical computation of the numerical dispersion relation is as follows. Given a computational mode with wave number $K \in(-\pi / \Delta x,+\pi / \Delta x]$, one first finds the uniquely defined wave number $k$ from $\mathrm{e}^{\mathrm{i} K \Delta x}=R^{1}(\mathrm{i} k \Delta x)$. The analytic dispersion relation $\mathcal{D}(\omega, k)=0$ implies a corresponding frequency $\omega$. One then uses $R^{0}(i \omega \Delta t)=\mathrm{e}^{\mathrm{i} \phi}$ to determine the uniquely defined phase $\phi(\omega \Delta t)$. Finally, the modulo operation (2.7) gives the computational frequency $\Omega$.

A number of important conclusions may be drawn. (i) Monotonicity of $\phi$ and $\theta$ implies $\phi^{\prime} \geq 0$, $\theta^{\prime} \geq 0$, and we obtain 
COROLlary 4. The numerical group velocity

$$
V_{g}=-\frac{\partial \mathcal{D}_{N}}{\partial K} / \frac{\partial \mathcal{D}_{N}}{\partial \Omega}
$$

at $(K, \Omega)$ has the same sign as the group velocity

$$
v_{g}=-\frac{\partial \mathcal{D}}{\partial k} / \frac{\partial \mathcal{D}}{\partial \omega}
$$

for the associated pair $(k, \omega)$.

(ii) Monotonicity of $\phi$ and $\theta$ implies that we can define the inverses of $\phi$ and $\theta$ and the two explicit relations

$$
\omega=\frac{1}{\Delta t} \phi^{-1}(\Delta t \Omega), \quad k=\frac{1}{\Delta x} \theta^{-1}(\Delta x K) .
$$

Using these two relations, we find that a numerical single mode solution satisfies

$$
\overline{\mathbf{a}}^{T}(i \omega \mathbf{K}+i k \mathbf{L}-\mathbf{A}) \mathbf{a}=0 .
$$

Definition (3.7) for the energy density and flux implies now

COROLlary 5. The energy density and flux, as defined in (3.7), satisfy

$$
F_{N}=V_{g} E_{N}
$$

for a numerical single mode solution, where $V_{g}$ is the numerical group velocity of the multisymplectic scheme.

(iii) Because of (2.7), non-intersecting branches of the analytic dispersion relation may intersect in the $(K, \Omega)$ plane unless either the implicit midpoint method is applied in time or $\Delta t$ is chosen such that

$$
\sup _{k \in S}|\phi(\omega(k) \Delta t)| \leq \pi
$$

where $k$ is to be restricted to the proper domain $S$ determined by the possible numerical wave numbers $-\pi<K \Delta x \leq \pi$. Intersecting branches (resonances) of the numerical dispersion relation may lead to instabilities of the method under small perturbations (see [2] for a discussion of the analytic case). Exclusion of intersecting branches leads to a CFL-type step-size restriction (except for an implicit midpoint time discretization). This will be discussed further in the following section.

5. An example: Fourth-order GL RK method in space and time. The linear wave equation

$$
u_{t t}=u_{x x}
$$

can be reformulated as a multi-symplectic PDE of the form

$$
-v_{t}-w_{x}=0, \quad u_{t}=v, \quad u_{x}=-w
$$

with $\mathbf{z}=(u, v, w)^{T}$,

$$
\mathbf{K}=\left[\begin{array}{ccc}
0 & -1 & 0 \\
1 & 0 & 0 \\
0 & 0 & 0
\end{array}\right], \quad \mathbf{L}=\left[\begin{array}{ccc}
0 & 0 & -1 \\
0 & 0 & 0 \\
1 & 0 & 0
\end{array}\right], \quad \mathbf{A}=\left[\begin{array}{ccc}
0 & 0 & 0 \\
0 & 1 & 0 \\
0 & 0 & -1
\end{array}\right]
$$

The analytic dispersion relation is $\omega^{2}=k^{2}$. The application of the fourth-order GL RK method in space yields

$$
\tan \frac{K \Delta x}{2}=\frac{k \Delta x}{2-\frac{k^{2} \Delta x^{2}}{6}}
$$



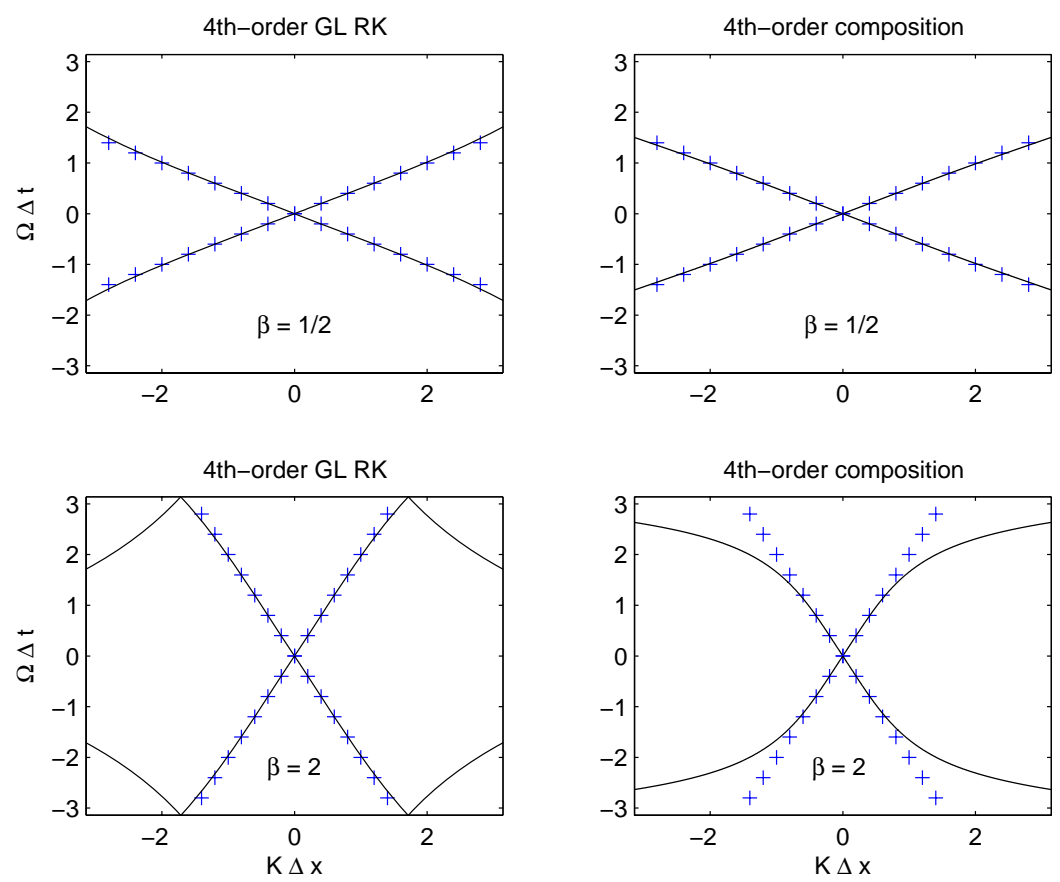

FIG. 5.1. The numerical dispersion relations displayed in the left two plots are for a fourth-order $G L R K$ space and time discretization for different values of $\beta=\Delta t / \Delta x$. The intersecting branches can clearly be seen for $\beta=2$. These intersecting branches are no longer presented in a fourth-order space and time discretization with the time discretization now being a fourth-order composition method based on the implicit midpoint method. These results are displayed in the two plots to the right.

and we find that $k \Delta x$ must be in the range of $0 \leq k \Delta x<\sqrt{12}$ for computational wave numbers $K$ satisfying $0 \leq K \Delta x<\pi$. More specifically, we obtain

$$
k \Delta x=-\frac{3}{b}+\sqrt{\frac{9}{b^{2}}+12}, \quad b=\tan \frac{K \Delta x}{2} .
$$

Let us apply the same fourth-order method as the temporal discretization. Upon introducing the step-size mesh ratio $\beta=\Delta t / \Delta x$, one finds that $\omega \Delta t$ satisfies the inequalities

$$
|\omega \Delta t|<\beta \sqrt{12} .
$$

Branches of the numerical dispersion relation intersect whenever $\beta>1$. However, $\phi^{\prime} \geq 0$ (i.e. preservation of the sign of the group velocity) is guaranteed for any value of $\beta$. See Fig. 5.1 for both cases $\beta<1$ and $\beta>1$.

Intersecting branches can be avoided altogether by an implicit midpoint discretization in time. Higher-order temporal discretization based upon the implicit midpoint method can be constructed using, e.g., Yoshida's fourth-order composition method [21]. The composed method still gives rise to a stability function of the form

$$
R(i \omega \Delta t)=\mathrm{e}^{\mathrm{i} \phi}, \quad \phi(\omega \Delta t)=2 \phi_{\mathrm{IM}}(\gamma \omega \Delta t)+\phi_{\mathrm{IM}}((1-2 \gamma) \omega \Delta t),
$$

where $\gamma=1 /\left(2-2^{1 / 3}\right)$ and $\phi_{\mathrm{IM}}$ is the phase function for the implicit midpoint method. It can be verified that $|\phi(\omega \Delta t)| \leq \pi$ and that $\phi$ is a monotone function. See Fig. 5.1 for numerical results.

6. The explicit midpoint scheme. This section discusses the effect of symplectic noncompact differencing methods by means of the explicit midpoint scheme. 
Applying the explicit midpoint rule in both space and time yields a simple second-order twostep method. Introducing

$$
\delta_{t}^{1 / 2} \mathbf{z}^{n, m}=\frac{1}{2 \Delta t}\left(\mathbf{z}^{n+1, m}-\mathbf{z}^{n-1, m}\right) \quad \text { and } \quad \delta_{x}^{1 / 2} \mathbf{z}^{n, m}=\frac{1}{2 \Delta x}\left(\mathbf{z}^{n, m+1}-\mathbf{z}^{n, m-1}\right),
$$

in order to simplify notation, we present the explicit midpoint scheme in the following proposition.

Proposition 2. The discretization

$$
\mathbf{K} \delta_{t}^{1 / 2} \mathbf{z}^{n, m}+\mathbf{L} \delta_{x}^{1 / 2} \mathbf{z}^{n, m}=\nabla_{\mathbf{z}} S\left(\mathbf{z}^{n, m}\right),
$$

satisfies a discrete multi-symplectic conservation law

$$
\delta_{t}^{+}\left(\mathbf{d} \mathbf{z}^{n, m} \wedge \mathbf{K} \mathbf{d z} \mathbf{z}^{n-1, m}\right)+\delta_{x}^{+}\left(\mathbf{d} \mathbf{z}^{n, m} \wedge \mathbf{L} \mathbf{d} \mathbf{z}^{n, m-1}\right)=0 .
$$

Proof. The associated variational equation is given by

$$
\mathbf{K} \delta_{t}^{1 / 2} \mathbf{d} \mathbf{z}^{n, m}+\mathbf{L} \delta_{x}^{1 / 2} \mathbf{d} \mathbf{z}^{n, m}=S_{z z}\left(\mathbf{z}^{n, m}\right) \mathbf{d} \mathbf{z}^{n, m} .
$$

After taking the wedge product with $\mathbf{d z}^{n, m}$, we get

$$
\mathbf{d} \mathbf{z}^{n, m} \wedge \mathbf{K} \delta_{t}^{1 / 2} \mathbf{d} \mathbf{z}^{n, m}+\mathbf{d} \mathbf{z}^{n, m} \wedge \mathbf{L} \delta_{x}^{1 / 2} \mathbf{d} \mathbf{z}^{n, m}=0,
$$

which is equivalent to

$$
\begin{aligned}
0= & \frac{1}{\Delta t}\left(\mathbf{d} \mathbf{z}^{n, m} \wedge \mathbf{K}\left(\mathbf{d} \mathbf{z}^{n+1, m}-\mathbf{d z}^{n-1, m}\right)\right) \\
& +\frac{1}{\Delta x}\left(\mathbf{d} \mathbf{z}^{n, m} \wedge \mathbf{L}\left(\mathbf{d z}^{n, m+1}-\mathbf{d} \mathbf{z}^{n, m-1}\right)\right)
\end{aligned}
$$

Since

$$
\begin{aligned}
\mathbf{d z}^{n, m} \wedge \mathbf{K}\left(\mathbf{d} \mathbf{z}^{n+1, m}-\mathbf{d} \mathbf{z}^{n-1, m}\right) & =\mathbf{d} \mathbf{z}^{n, m} \wedge \mathbf{K} \mathbf{d} \mathbf{z}^{n+1, m}-\mathbf{d} \mathbf{z}^{n, m} \wedge \mathbf{K} \mathbf{d} \mathbf{z}^{n-1, m} \\
& =\mathbf{d} \mathbf{z}^{n+1, m} \wedge \mathbf{K} \mathbf{d} \mathbf{z}^{n, m}-\mathbf{d} \mathbf{z}^{n, m} \wedge \mathbf{K} \mathbf{d} \mathbf{z}^{n-1, m}
\end{aligned}
$$

and a similar result for the spatial discretization part, this gives the desired result. $\square$

Applying this discretization to the linear equation yields

$$
\mathbf{K} \delta_{t}^{1 / 2} \mathbf{z}^{n, m}+\mathbf{L} \delta_{x}^{1 / 2} \mathbf{z}^{n, m}=\mathbf{A} \mathbf{z}^{n, m} .
$$

Lemma 1. Numerical solutions of the method (6.1) satisfy the numerical dispersion relation

$$
\mathcal{D}_{\mathbf{A}}(\omega, k)=0
$$

for $\mathcal{D}_{\mathbf{A}}$ as given in (1.4) with

$$
\omega=\frac{\sin (\Omega \Delta t)}{\Delta t} \quad \text { and } \quad k=\frac{\sin (K \Delta x)}{\Delta x},
$$

for $-\pi<\Delta t \Omega \leq \pi$ and $-\pi<\Delta x K \leq \pi$.

Proof. Substituting the (complex-valued) numerical solution ansatz

$$
\mathbf{z}^{n, m}=\mathrm{e}^{\mathrm{i}(\Omega n \Delta t+K m \Delta x)} \mathbf{a}
$$

into (6.1) gives

$$
\left(\mathbf{K} \frac{\mathrm{e}^{\mathrm{i} \Omega \Delta t}-\mathrm{e}^{-\mathrm{i} \Omega \Delta t}}{2 \Delta t}+\mathbf{L} \frac{\mathrm{e}^{\mathrm{i} K \Delta x}-\mathrm{e}^{-\mathrm{i} K \Delta x}}{2 \Delta x}-\mathbf{A}\right) \mathbf{a}=0 .
$$


Now, using the trigonometric identities

$$
\mathrm{e}^{\mathrm{i} \Omega \Delta t}-\mathrm{e}^{-\mathrm{i} \Omega \Delta t}=2 \mathrm{i} \sin (\Delta t \Omega), \quad \text { and } \quad \mathrm{e}^{\mathrm{i} K \Delta x}-\mathrm{e}^{-\mathrm{i} K \Delta x}=2 \mathrm{i} \sin (\Delta x K),
$$

the proof is completed. by

A first important conclusion is that the relation $R(\mathrm{i} \omega \Delta t)=\mathrm{e}^{\mathrm{i} \phi}$ implies that $\phi(\omega \Delta t)$, defined

$$
\sin \phi=\omega \Delta t,
$$

is no longer monotone and is not defined for $|\omega \Delta t|>1$ (instability of the method). Furthermore, the non-monotonicity implies the existence of spurious roots in the numerical dispersion relation. A similar observation applies to the midpoint spatial discretization. Non-monotonicity also implies that the sign of the numerical group velocity can be different from the sign of the analytic value for the corresponding $(\omega, k)$ pair.

We now investigate the behavior of the explicit midpoint space-time discretization for the linear wave equation (5.1). The discretization can be derived from the discrete Lagrangian

$$
L=\frac{1}{2} \sum_{n, m}\left[\left(\frac{u^{n+1, m}-u^{n-1, m}}{2 \Delta t}\right)^{2}+\left(\frac{u^{n, m+1}-u^{n, m-1}}{2 \Delta x}\right)^{2}\right]
$$

following the principle of discrete variational mechanics [15]. The numerical dispersion relation is given by

$$
\sin ^{2}(\Omega \Delta t)=\frac{\Delta t^{2}}{\Delta x^{2}} \sin ^{2}(K \Delta x)
$$

and is real-valued provided $\Delta t / \Delta x \leq 1$ (CFL condition). However, even for $\Delta t / \Delta x \leq 1$, the numerical dispersion relation has spurious roots for given $K$ and $\Omega$, respectively.

Let us first demonstrate the implication of the non-monotone numerical dispersion relation for solutions of the linear wave equation (5.1). We solve the wave equation with the explicit midpoint method in space and time over a spatial domain $x \in[0,1)$ with periodic boundary conditions and initial data

$$
u(x, 0)=f(x), \quad v(x, 0)=-f^{\prime}(x), \quad f(x)=\exp \left(-30^{2}(x-1 / 2)^{2}\right) \sin (50 \pi x) .
$$

The analytic solution consists of a wave packet moving to the right. The numerical solution for $\Delta x=1 / 450$ and $\Delta t=0.9 \Delta x$ is displayed in Fig. 6.1. The small perturbation moving towards the left is due to the non-monotone numerical dispersion relation and would be absent for a GL RK discretization in space and time.

The spurious roots for $K$ and/or $\Omega$ in the numerical dispersion relation of the space-time explicit midpoint discretization can be partially eliminated by substituting a symplectic one-step method in space or in time. As an example we consider the symplectic leapfrog/Störmer-Verlet (LF/SV) method. Applying the LF/SV method in space yields the numerical dispersion relation

$$
\sin ^{2}(\Omega \Delta t)=\frac{4 \Delta t^{2}}{\Delta x^{2}} \sin ^{2}(K \Delta x / 2),
$$

while application in time implies

$$
\sin ^{2}(\Omega \Delta t / 2)=\frac{\Delta t^{2}}{4 \Delta x^{2}} \sin ^{2}(K \Delta x) .
$$

As shown in Fig. 6.2 both combinations still imply spurious roots in their numerical dispersion relations. The same statement is true if the Störmer-Verlet method is replace in time by the implicit midpoint method. The only difference is that there is no longer a stability restriction on $\beta$. See Fig. 6.3.

It should be noted that there are spatial multi-step discretizations with monotone phase function [9]. However those methods do not seem to satisfy a discrete variational principle and, hence, do not give rise to multi-symplectic discretization methods. 

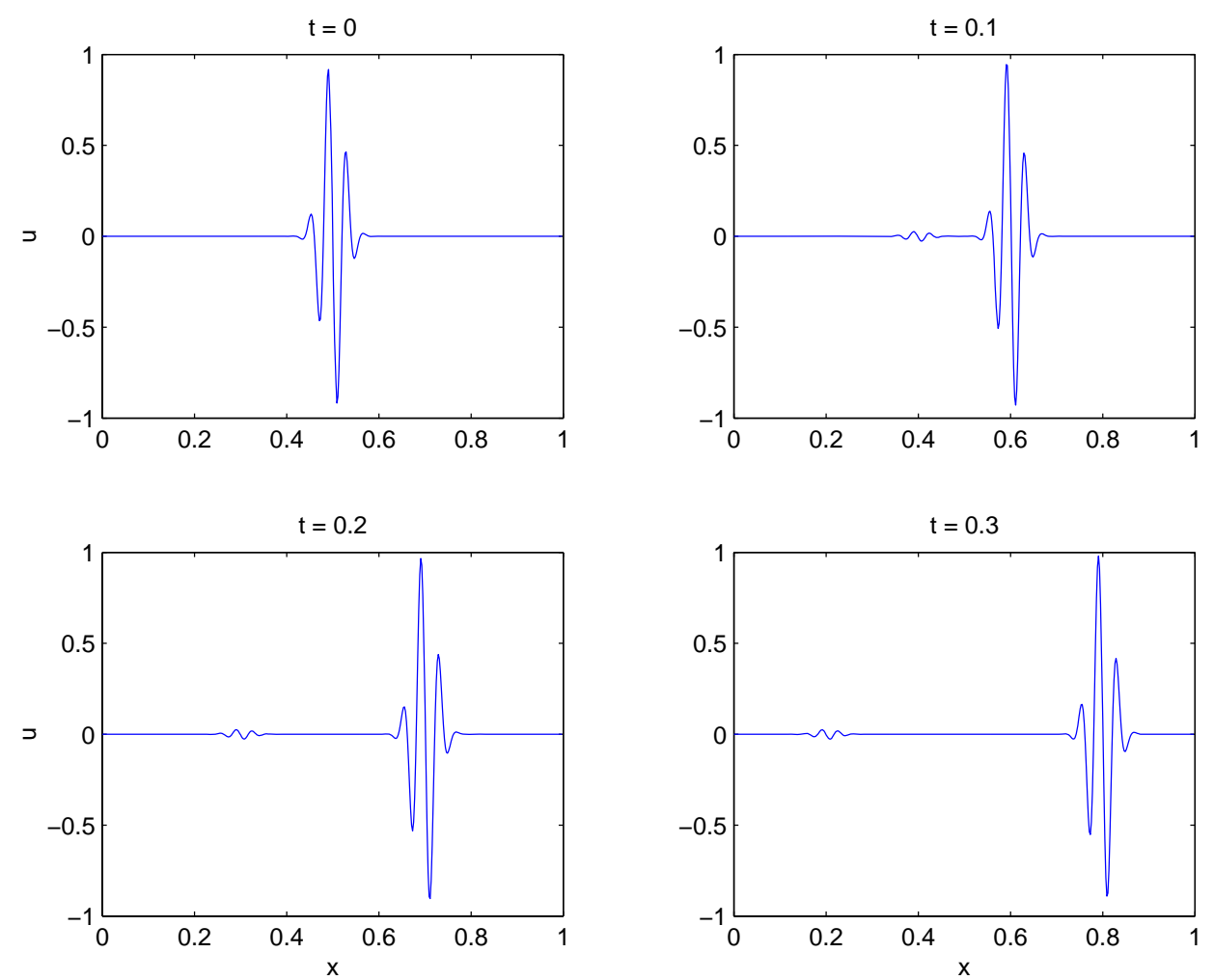

FIG. 6.1. Numerical propagation of a wave packet under the space-time explicit midpoint (EMP) method. The analytic solution consists of a wave packet moving entirely to the right. The displayed small perturbation moving towards the left is a numerical artifact due to the non-monotone numerical dispersion relation of the EMP method.
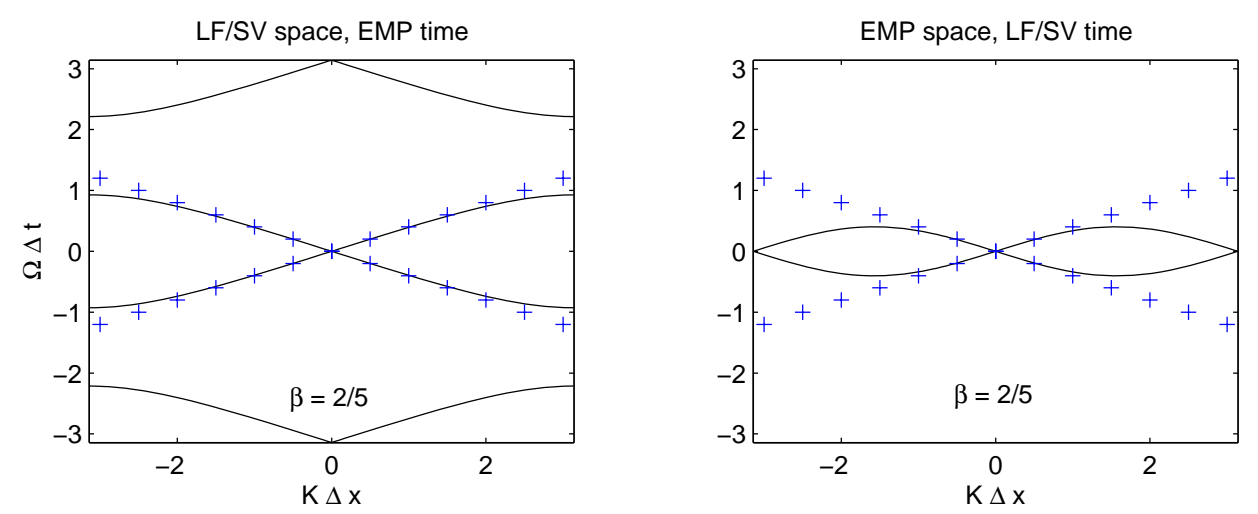

FIG. 6.2. The numerical dispersion relations displayed on the left is for an explicit midpoint (EMP) discretization in time and a Störmer-Verlet $(L F / S V)$ discretization in space. The spurious branches (roots) in the numerical dispersion relation are due to the multi-step EMP method. On the contrary, an LV/SV discretization in time combined with a EMP discretization in space yields a non-monotone dispersion relation implying spurious roots.

7. Summary: Towards a characterization of multi-symplectic integration methods. There are a variety of numerical discretizations that satisfy a discrete multi-symplectic conservation law. When applied to linear multi-symplectic PDEs with wave-like solutions, all these methods yield real-valued numerical dispersion relations

$$
\mathcal{D}_{N}(\Omega, K)=0
$$



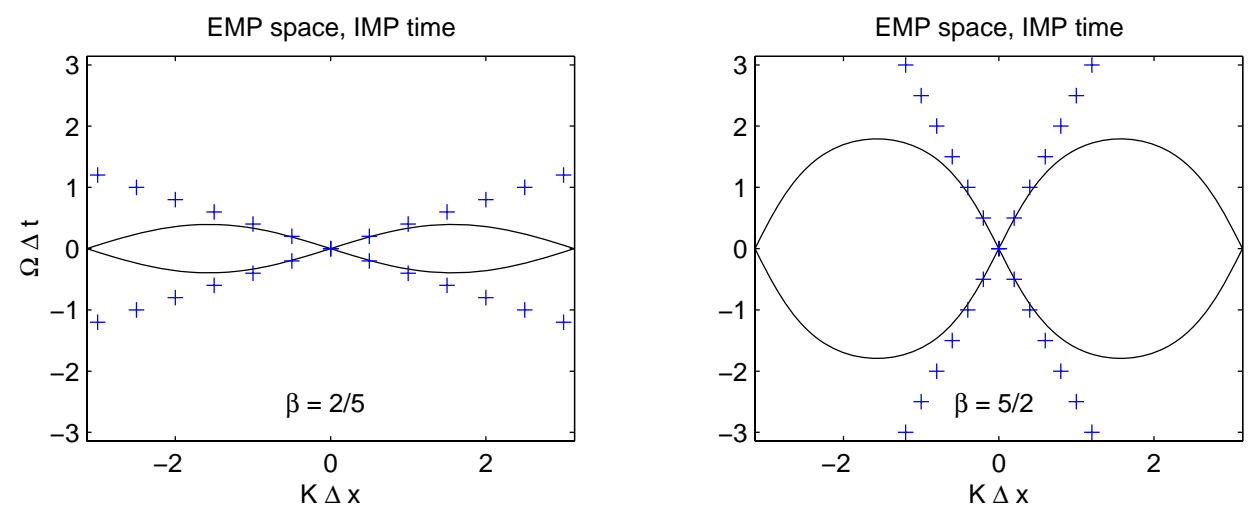

FIG. 6.3. The displayed numerical dispersion relations are for an explicit midpoint (EMP) discretization in space and an implicit midpoint (IMP) discretization in time. Results are shown for $\beta<1$ and $\beta>1$.

provided the ratio $\Delta t / \Delta x$ satisfies a CFL-type condition $\Delta t / \Delta x \leq C$, where $C$ is a positive number. This follows from the fact that $R(z)$, i.e. $\mathbf{z}^{n+1}=R(z) \mathbf{z}^{n}$, of a (stable and consistent) symplectic (one-step or multi-step) method has to satisfy

$$
|R(\mathrm{i} \sigma)|=1
$$

for sufficiently small $\sigma \geq 0$ because of conservation of volume.

One-step multi-symplectic discretizations in space and time, such as GL RK, lead to (complexvalued) numerical approximations of the form

$$
\mathbf{z}^{n, m}=R^{0}(\mathbf{i} \omega \Delta t)^{n} R^{1}(\mathbf{i} k \Delta x)^{m} \mathbf{a},
$$

where $R^{0}(z)$ and $R^{1}(z)$ are the stability functions for the temporal and spatial discretization, respectively. It follows that multi-symplectic GL RK discretizations in space and time are unconditionally stable, i.e. $C=\infty$.

Another key aspect of numerical methods is the existence or non-existence of spurious roots in the numerical dispersion relation. The existence of spurious modes can be excluded provided (i)

$$
R^{0}(\mathrm{i} y)=\mathrm{e}^{\mathrm{i} \phi(y)}, \quad R^{1}(\mathrm{i} y)=\mathrm{e}^{\mathrm{i} \theta(y)}
$$

for monotone phase functions $\phi, \theta$ and (ii) $\Delta t / \Delta x$ satisfies a CFL-type condition $\Delta t / \Delta x \leq c$. While all GL RK methods have monotone phase functions [10], the implicit midpoint time discretization is the only GL RK method for which $c=\infty$. Monotonicity of the phase function $\phi(\sigma)$ alone implies conservation of the sign of the group velocity under numerical discretization. Hence wave packets propagate numerically at a perturbed speed but in the correct direction. A multi-symplectic discretization with

$$
c<\frac{\Delta t}{\Delta x} \leq C
$$

will, in general, lead to numerically induced 1-1 resonances (intersections of solution branches of the dispersion relation). Those spurious resonances can lead to instabilities of the method under small perturbations in the same manner as analytic resonances can give rise to instabilities [2]. It was found that higher-order time discretizations with $c=\infty$ can be constructed by proper composition of the implicit midpoint method. It should be noted that multi-symplectic methods can be subject to additional numerically induced resonances. (Compare, e.g., Mandziuk and Schlick [14] for the case of Hamiltonian ODEs.) This issue will be investigated in detail in a forthcoming publication. 
Acknowledgements The authors thank an anonymous referee for suggestions that led to a more thorough treatment of the energy transport under multi-symplectic discretization methods.

\section{REFERENCES}

[1] U. M. Ascher and R. I. McLachlan. Multisymplectic box schemes and the Korteweg-de Vries equation. Appl. Numer. Math., 48:255-269, 2004.

[2] T. J. Bridges. A geometric formulation of the conservation of wave action and its implications for signature and the classification of instabilities. Proceedings of the Royal Society London, 453(A):1365-1395, 1997.

[3] T. J. Bridges. Multi-symplectic structures and wave propagation. Mathematical Proceedings of the Cambridge Philosophical Society, 121:147-190, 1997.

[4] T. J. Bridges and G. Derks. Unstable eigenvalues and the linearization about solitary waves and fronts with symmetry. Proceedings of the Royal Society London, 455(A):2427-2469, 1999.

[5] T. J. Bridges and G. Derks. Linear instability of solitary wave solutions of the Kawahara equation and its generalizations. SIAM Journal of Mathematical Analysis, 33:1356-1378, 2002.

[6] T. J. Bridges, G. Derks, and G. Gottwald. Stability and instability of solitary waves of the fifth-order KdV equation: a numerical framework. Physica D, 172:190-216, 2002.

[7] T. J. Bridges and S. Reich. Multi-symplectic integrators: Numerical schemes for Hamiltonian PDEs that conserve symplecticity. Phys. Lett. A, 284:184-193, 2001.

[8] T. J. Bridges and S. Reich. Multi-symplectic spectral discretizations for the Zakharov-Kuznetsov and shallowwater equations. Physica D, 152:491-504, 2001.

[9] J. Frank and S. Reich. On spurious reflections, nonuniform grids and finite difference discretizations of wave equations. 2005. in preparation.

[10] N. Guglielmi and E. Hairer. Order starts and stability for delay differential equations. Numerische Mathematik, 83:371-383, 1999.

[11] E. Hairer, Ch. Lubich, and G. Wanner. Geometric Numerical Integration: Structure Preserving Algorithms for Ordinary Differential Equations. Springer-Verlag, Berlin, 2002.

[12] S. Kournabaeva and S. Shkoller. A variational approach to second-order multisymplectic field theories. $J$. Geom. Phys., 35:333-366, 2000.

[13] B. Leimkuhler and S. Reich. Simulating Hamiltonian Dynamics. Cambridge University Press, Cambridge, 2005.

[14] M. Mandziuk and T. Schlick. Resonance in the dynamics of chemical systems simulated by the implicitmidpoint scheme. Chem. Phys. Lett., 237:525-535, 1995.

[15] J. E. Marsden, G. P. Patrick, and S. Shkoller. Multi-symplectic geometry, variational integrators, and nonlinear PDEs. Communications in Mathematical Physics, 199:351-395, 1999.

[16] R. I. McLachlan. Symplectic integration of Hamiltonian wave equations. Numerische Mathematik, 66:465-492, 1994.

[17] B. E. Moore. A modified equations approach for multi-symplectic integration methods. 2003. PhD thesis, University of Surrey.

[18] B. E. Moore and S. Reich. Multi-symplectic integrators for Hamiltonian PDEs. Future Generation Computer Systems, 19:395-402, 2003.

[19] S. Reich. Multi-symplectic Runge-Kutta collocation methods for Hamiltonian wave equations. Journal of Computational Physics, 157:473-499, 2000.

[20] G. B. Whitham. Linear and Nonlinear Waves. Wiley-Interscience, New York, 1974.

[21] H. Yoshida. Construction of higher order symplectic integrators. Phys. Lett. A, 150:262-268, 1990. 